\title{
LA CIENCIA DEL DERECHO PROCESAL CONSTITUCIONAL. ESTUDIOS EN HOMENAJE A HÉCTOR FIX-ZAMUDIO EN SUS CINCUENTA AÑOS COMO INVESTIGADOR DEL DERECHO
}

Ed. CECOCH, IIJ de la UNAM y Editorial Librotecnia, Santiago de Chile, 2009

Humberto Nogueira Alcalá

Esta publicación nacional se debe a la voluntad de que los artículos chilenos de la obra en homenaje a Héctor Fix- Zamudio en sus cincuenta años como investigador del derecho, que lleva por título "La ciencia del derecho procesal constitucional", coordinado por Eduardo Ferrer Mac-Gregor y Arturo Zaldívar Lelo de Larrea, pudiera ser socializada en Chile, ya que no era posible que la obra completa de doce tomos, editados en M éxico, por las casas editoras Porrúa, Marcial Pons y el Instituto M exicano de Derecho Procesal Constitucional, aparecido en el segundo semestre de 2008, pudiera circular por su alto costo.

El homenaje al maestro Fix Zamudio es un reconocimiento a su señero aporte académico en el ámbito del derecho público, el cual se inició con sus trabajos en el ámbito del derecho procesal, siendo discípulo de Niceto Alcalá-Zamora y Castillo quien codirigió su tesis de grado en derecho, destacando en este ámbito sus obras sobre "Veinticinco años de evolución de la justicia constitucional (1940-1965)" (M éxico, UNAM, 1968); "La protección jurídica y procesal de los derechos humanos ante las jurisdicciones nacionales" (Madrid, Civitas, 1982); junto a muchas otras obras dentro de las cuales pueden mencionarse "Ensayos sobre el derecho de amparo" (México, UNAM - Porrúa, varias ediciones); "Justicia Constitucional, ombudsman y derechos humanos" (M éxico, $\mathrm{CNDH}, 1993) ;$ para luego incursionar en el derecho constitucional, tanto mexicano como comparado, donde destaca su obra "Los tribunales constitucionales y los derechos humanos" (M éxico, Porrúa, 1985); "Problemas contemporáneos del Poder Judicial" (M éxiCo, UNAM , 1986); "Introducción al estudios de la defensa de la Constitución en el ordenamiento mexicano" (M éxico, UNAM, varias ediciones); junto a José Ramón Cossío "El poder judicial en el ordenamiento mexicano" (M éxico, FCE, 1996); junto a su hijo Héctor Fix Fierro, "El Consejo de la Judicatura" (M éxico, Unam, 1996); junto a Salvador Valencia 
Carmona, "Derecho constitucional mexicano y comparado" (M éxico, Porrúa, varias ediciones); siendo en el ámbito latinoamericano, quien desarrolló y ha contribuido con mayor energía a la conceptualización y desarrollo dogmático del derecho procesal constitucional en la región, donde destaca su obra el "El juicio de amparo" (M éxico, Porúa, 1964); "Latinoamérica. Constitución, proceso y derechos humanos" (México, Porrúa, 1988); "Introducción a la defensa de la Constitución en el ordenamiento mexicano" (M éxico, UNAM, varias ediciones), sin perjuicio de sus aportes conceptuales y al perfilamiento del derecho procesal constitucional como disciplina, que ya había iniciado entre 1955 y 1956, en "La garantía jurisdiccional de la Constitución mexicana. Ensayo de una estructuración procesal del amparo", redactado en 1955 y publicado en diversas revistas mexicanas en 1956.

Su gran obra académica la desarrolla como investigador del Instituto de Investigaciones Jurídicas de la UNAM, del cual fue director por doce años, entre 1966 y 1978, durante cuya dirección el Instituto se desarrolló y adquirió el rigor científico y disciplinario que lo ha convertido en la institución líder en la materia de América Latina, el cual publica más de cien obras anualmente de sus investigadores, del cual hoy el Maestro Fix-Zamudio es investigador emérito nombrado por el Consejo Universitario desde el año 1987.

Fuera de sus importantes investigaciones y publicaciones en el ámbito del derecho procesal y del derecho constitucional tanto mexicano como comparado, su principal aporte al derecho en una perspectiva regional ha sido el desarrollo del derecho procesal constitucional.

\subsection{EL USO DEL VOCABLO POR NICETO ALCALÁ ZAMORA Y CASTLLLO}

El uso del concepto de derecho procesal constitucional surge en la década del 40 del siglo XX, siendo el jurista y procesalista español Niceto A Icalá Zamora y Castillo su creador, en sus obras de exilio en Argentina y México.

El vocablo fue utilizado por primera vez por Niceto A Icalá Zamora y Castillo, en su etapa de exilio en Argentina, el cual lo utiliza en su libro "Ensayos de derecho procesal (civil, penal y constitucional)", publicado en Buenos Aires en 1944, reiterándolo en artículo publicado en la Revista de Derecho Procesal, editada en Buenos Aires por Hugo Alsina (año III, 2ª parte, 1945, p. 77).

Niceto A lcalá Zamora y Castillo luego emigra a M éxico contratado por la Universidad Nacional Autónoma de M éxico, donde desarrolla su obra Proceso, autocomposición y autodefensa, ${ }^{1}$ en la cual expresa su opinión de que Kelsen constituye el funda-

1 Alcalá-Zamora y Castilo, Niceto. Proceso, autocomposición y autodefensa (contribución a los fines del proceso), Primera edición, M éxico, 1947. 
dor del derecho procesal constitucional. ${ }^{2}$ Es allí, en Ciudad de M éxico y en la UNAM que se encuentra Alcalá Zamora y Castillo con el joven Héctor Fix-Zamudio, a quien guiará en el desarrollo de su tesis de licenciatura en derecho de 1955.

\subsection{LOS ANTECEDENTES DOCTRINALES DEL DERECHO PROCESAL CONSTITUCIONAL}

Puede señalarse que en la doctrina fueron Hans Kelsen, Eduardo Couture, Piero Calamandrei y Mauro Cappeletti los que aportaron las bases para el nacimiento del derecho procesal constitucional.

Ya Niceto A lcalá Zamora y Castillo sostiene en su obra "Proceso, autocomposición y autodefensa (contribución a los fines del proceso)" que Kelsen constituye el fundador del derecho procesal constitucional (3a ed. M éxico, UNAM, 1991, p. 215); lo que es también asumido por Fix-Zamudio en su memoria de licenciado en la conclusión quinta de ella. Sin lugar a dudas, para ello se considera que fue integrante del equipo que elaboró el texto referente al Tribunal Constitucional austriaco incorporado en la Constitución de 1920, del cual fue magistrado entre 1921 y 1930, luego que se dictara y promulgara el 13 de junio de 1921, la Ley Federal sobre organización del Tribunal Constitucional, sin perjuicio de mantenerse en la cátedra universitaria en la Facultad de Derecho de Viena.

Hans Kelsen es el autor del primer estudio sistemático sobre la jurisdicción constitucional desarrollado en 1928 en su obra "La garantie jurisdictionelle de la Constitution (La justice constitutionnelle)", ${ }^{3}$ donde fundamenta y desarrolla en forma sistemática la existencia de una jurisdicción constitucional concentrada y especializada.

Eduardo Couture se refiere al derecho procesal constitucional en su trabajo "Las garantías constitucionales del proceso civil", publicado en el libro "Estudios de Derecho Procesal en Honor de Hugo Alsina" (Buenos Aires, 1946, pp. 158-173), como asimismo en su obra clásica "Fundamentos del Derecho Procesal Civil", cuya primera edición es de 1947, donde dedica la tercera parte del tomo I a los casos del Derecho Procesal Constitucional. A simismo, se le considera el padre del Derecho Constitucional Procesal, el cual comprende el estudio de las garantías constitucionales de acceso a la jurisdicción o tutela judicial efectiva y las reglas sustantivas y adjetivas del debido proceso.

Piero Calamandrei, distinguido procesalista y luego profesor de derecho constitucional italiano en la postguerra, realizará aportaciones significativas al desarrollo del dere-

Alcalá-Zamora y Castillo, Niceto. Proceso, autocomposición y autodefensa (contribución a los fines del proceso). 3a ed., México, UNAM, 1991, p. 215.

3 Ver en Revue de Droit Public en France et a l'Étranger, Paris, 1928, pp. 52-143. Traducido al español por Rolando Tamayo y Salmorán, "La garantía jurisdiccional de la Constitución (La justicia Constitucional)", en Anuario Jurídico, I, UNAM, México, 1974, pp. 471-515. 
cho procesal constitucional al establecer los fundamentos de la jurisdicción constitucional italiana en su obra La Illegittimita constituzionale delle leggi nel processo civile, ${ }^{4}$ la cual fue posteriormente, en 1962, traducida al español. ${ }^{5}$ A simismo, el autor aborda la materia en tres artículos: "Potere Giudiziario e Suprema Corte Costituzionale", "La Illegittimita constituzionale delle leggi nel processo civile", y "Corte Costituzionale e autoritá giudiziaria", publicados en Opere Giudiriche (Napoli, 1968, tomo III, pp. 215-225; 337-412; y 609-654), los cuales son traducidos al español por Sentís M elendo. ${ }^{6}$

M auro Cappelletti, por su parte, aportará en el ámbito de la jurisdicción constitucional, que esta no sólo se refiere al control constitucional orgánico, sino que se amplía con la jurisdicción constitucional protectora de los derechos fundamentales o la jurisdicción constitucional de las libertades, lo que precisa en su obra La giurisdizione costituzionale delle liberta (Giuffrè, M ilano, 1955), la que será traducida al español por el propio Fix-Zamudio como La jurisdicción constitucional de la libertad (con referencia a los ordenamientos alemán, suizo y austriaco). ${ }^{7}$ Además el maestro italiano agrega el necesario estudio del derecho procesal trasnacional o Jurisdicción constitucional trasnacional, ${ }^{8}$ materia que trata también en su artículo "Justicia Constitucional supranacional", traducido por Luis Dorantes Tamayo y publicado en la Revista de la Facultad de Derecho de M éxico N 110 , mayo-agosto, Tomo XXVIII, M éxico, 1978, pp. 337 y ss.

El desarrollo del derecho procesal constitucional como disciplina jurídica y su estudio sistemático por Héctor Fix-Zamudio.

Es, sin duda, el maestro Héctor Fix-Zamudio, discípulo de A lcalá Zamora y Castillo, quien desarrollará y sistematizará el derecho procesal constitucional como disciplina jurídica, cuyo primer trabajo fue su tesis de licenciado en derecho en 1955, denominada "La garantía jurisdiccional de la Constitución mexicana (ensayo de una estructuración procesal del amparo)", Facultad de Derecho, UNAM , 1955, 180 páginas, defendida en enero de 1956; publicados dos de sus capítulos en la Revista La Justicia en $1956^{9}$ y luego el contenido completo en su obra "El juicio de Amparo", Editorial Porrúa, M éxico, 1964, pp. 5-70. Asimismo, en 1956, publica el artículo "La aportación de Piero Calamandrei al Derecho Procesal Constitucional" (Revista de la Facultad de Derecho de México, Tomo VI, N²4, octubre-diciembre de 1956).

4 Obra editada por CEDAM, Padova, Italia, 1950.

5 Incorporada al texto de CalamandRel Estudios sobre el proceso civil, Buenos Aires, 1962, pp. 21-120.

6 Calamandrei, Piero. Estudios sobre el proceso civil. Traducción de Sentís M elendo, Ejea, pp. 23-120 y 121-198.

7 CAPPELLETI, M auro. La jurisdicción constitucional de la libertad (con referencia a los ordenamientos alemán, suizo y austriaco). Instituto de Derecho Comparado-UNAM. Imprenta Universitaria, México, 1961.

8 Cappeletr, Mauro. Necesidad y legitimidad de la justicia constitucional, en Tribunales constitucionales europeos y derechos fundamentales, Madrid, Centro de Estudios Constitucionales, 1984, pp. 599-662.

9 El capítulo "El Derecho Procesal Constitucional", en La Justicia, Tomo XXVII, N 309-310, enero- febrero, 1956, pp. 12300-12313 y 12361-12364; y el capítulo "El Proceso Constitucional", en La Justicia, Tomo XXVII, N 317, septiembre de 1956, pp. 12625-12336. 
Ya en su tesis de licenciatura, Fix-Zamudio consideraba la existencia de "una disciplina instrumental que se ocupa del estudio de las normas que sirven de medio para la realización de las disposiciones contenidas en los preceptos constitucionales, cuando estos son desconocidos, violados o existe incertidumbre sobre su significado; siendo esta materia una de las ramas más jóvenes de la Ciencia del Derecho Procesal, y por lo tanto, no ha sido objeto todavía de una doctrina sistemática que defina su verdadera naturaleza y establezca sus límites dentro del inmenso campo del Derecho" ${ }^{10}$

Fix-Zamudio, en La garantía jurisdiccional de la Constitución mexicana (Ensayo de una estructura procesal del Amparo), se refiere al derecho procesal constitucional como aquel que se ocupa del examen de las garantías de la propia Ley Fundamental, y que están establecidas en el texto mismo de la norma suprema (p. 91).

Los aportes más maduros de Fix-Zamudio sobre la materia se encuentran en su obra Introducción al Derecho Procesal Constitucional que se publicara también por FUNDAP, en Querétaro, en el año 2002, como asimismo en sus obras Derecho constitucional mexicano y comparado (2003); y “La defensa de la Constitución en el ordenamiento mexicano“ (Ed. Porrúa, M éxico, 2006).

Fix-Zamudio en La garantía jurisdiccional de la Constitución mexicana (Ensayo de una estructura procesal del Amparo), conceptualiza el proceso constitucional como "conjunto armónico y ordenado de actos jurídicos, en vista de la composición de la litis de trascendencia jurídica, que establece una relación de las partes con el juzgador y que se desenvuelve en una serie concatenada de situaciones". ${ }^{11}$

En tal sentido, el Maestro Fix-Zamudio distingue el derecho procesal constitucional del derecho constitucional procesal, este último iniciado por Eduardo Couture, el cual se centra en el estudio y análisis de la normativa constitucional que regula el derecho a la jurisdicción y al debido proceso.

En Introducción al derecho procesal constitucional, Fix-Zamudio vuelve sobre el concepto de derecho procesal constitucional, señalando que "tiene por objeto el análisis científico, desde la perspectiva de la teoría o doctrina general del proceso o del Derecho Procesal, de las garantías constitucionales establecidas por la Carta Federal de 1917, con sus numerosas reformas posteriores". ${ }^{12}$ En la misma obra, el autor precisa que "[... ] debemos estar conscientes que hay una presencia de las diversas disciplinas procesales respecto del derecho sustantivo que es relativamente reciente y que ese desprendimiento ha sido paulatino, comenzando por el derecho procesal civil y

10 Fix-Zamudio, Héctor. La garantía jurisdiccional de la Constitución mexicana (Ensayo de una estructura procesal del Amparo), UNAM, México, D.F., 1955, p. 57.

11 Fix-Zamudio, Héctor. La garantía jurisdiccional de la Constitución mexicana (Ensayo de una estructura procesal del Amparo), UNAM, México, D.F., 1955, p. 106.

12 Fix-Zamudio, Héctor. Introducción al Derecho Procesal Constitucional. Ed. FUNDAp, Querétaro, 2002, pp. 113-114. 
penal, y sólo posteriormente los derechos procesales administrativos, de trabajo, agrario, etc. y una de las ramas más modernas, sino es que debe considerarse la más actual, es precisamente el derecho procesal constitucional". ${ }^{13}$

Finalmente, Fix-Zamudio en sus "Breves reflexiones sobre el concepto y contenido del derecho procesal constitucional", nos señala que el derecho procesal constitucional puede describirse "como la disciplina jurídica, situada dentro del campo del derecho procesal, que se ocupa del estudio sistemático de las instituciones y de los órganos por medio de los cuales pueden resolverse los conflictos relativos a la aplicación de los principios, valores y disposiciones fundamentales, con el objeto de reparar la violación de los mismos. [...] Esta rama del derecho procesal general tiene como contenido el análisis de las tres categorías que integran lo que se ha calificado como 'trilogía estructural del proceso', es decir, la acción, la jurisdicción y el proceso. Pero estas tres categorías esenciales poseen aspectos peculiares en el derecho procesal constitucional, y con este motivo, como un ensayo de sistematización de la materia de esta disciplina reciente, todavía en formación, adoptamos la terminología, ya acreditada, del notable procesalista italiano Mauro Cappelletti, quien además ha sido uno de los juristas que mayores aportaciones han hecho a este sector del derecho procesal" ${ }^{14}$

Estas últimas consideraciones de Fix-Zamudio deben ser moduladas, a que podemos sostener con Häberle y Landa que el derecho procesal constitucional es también un derecho constitucional concretizado, "no se trata sólo de aplicar la Constitución en función de las normas procedimentales, sino de darle a dichas normas un contenido propio, conforme a la Constitución, pues si bien el derecho procesal constitucional también requiere partir y remitirse a los principios generales del Derecho procesal, ello será posible en la medida que este último sea afín con los principios y valores constitucionales". ${ }^{15}$

Con Zagrebelsky, puede señalarse que la jurisdicción constitucional y los procedimientos de aplicación de la Constitución para la resolución de casos controvertidos lleva aparejada una teoría de la Constitución como norma sustancial, cada concepción de la Constitución lleva aparejado una concretización del procedimiento, así como cada concepción del procedimiento lleva aparejada una concepción de la Constitución. ${ }^{16}$ El derecho procesal constitucional es "sui generis", ya que comprende en sí pluralidad de perspectivas, que deben reconstruirse alrededor de bienes jurídicos múltiples. Se trata de un derecho procesal capaz de comprender las razones no siempre

13 Fix-Zamudio, Héctor. Introducción al Derecho Procesal Constitucional. Ed. FUNDAp, Querétaro, 2002, pp. 19 y ss.

14 Fix-ZAMUDIO, Héctor. "Breves reflexiones sobre el concepto y contenido del derecho procesal constitucional", en Ferrer Mac-Gregor, Eduardo. Derecho procesal constitucional. Tomo I. Tercera edición, Ed. Porrúa, México, 2003, p. 197.

15 LANDA, César. "Autonomía procesal del Tribunal Constitucional", en Revista Justicia Constitucional. Año $2 \mathrm{~N}^{\circ} 4$, julio-diciembre, 2006, Ed. Palestra, Lima, 2006, p. 67.

16 Zagrebelsky, Gustavo. ¿Derecho Procesal Constitucional? y otros ensayos de justicia constitucional. Ed. FUNDAp, México, 2004, pp. 17-18. 
coincidentes de la tutela subjetiva de los derechos fundamentales, pero también las razones de la tutela objetiva de la Constitución. ${ }^{17}$

El derecho procesal constitucional implica la existencia de determinadas instituciones, valores, principios, derechos y garantías constitucionales que se operacionalizan litigiosamente en procesos y procedimientos que regula la disciplina, los cuales implican la confluencia aplicativa de principios e instituciones tanto de derecho constitucional como de derecho procesal con particularidades propias de los procesos constitucionales. Es una disciplina sustantiva en la medida que tiene por objeto de estudio la defensa de la Constitución y la interpretación de la Carta Fundamental y los derechos fundamentales por las jurisdicciones constitucionales; estudia los órganos constitucionales, su organización, funciones y atribuciones; y es una disciplina adjetiva en la medida que utiliza principios y reglas procesales, aún cuando con peculiaridades propias de los procesos constitucionales, en especial, la legitimación, las partes, el concepto de cosa juzgada, relativización, principio de congruencia, los tipos de sentencia, la fuerza vinculante de la sentencia constitucional, entre otras materias.

Así, esta novel disciplina jurídica reconoce elementos y se alimenta de la dogmática constitucional y procesal en una interacción en la cual ninguno de ellos somete 0 anula al otro, reconociendo en la Constitución su derecho sustantivo y en el derecho procesal el derecho adjetivo. Además debe tenerse presente que el objeto que está en litigio es estrictamente una materia constitucional (la defensa y garantía de los derechos fundamentales y el control del poder político en el Estado Constitucional democrático haciendo efectiva la supremacía constitucional).

Durante las últimas dos décadas del siglo XX y esta primera década del siglo XXI, puede sostenerse que se ha desarrollado y expandido el interés académico por el derecho procesal constitucional, como asimismo se ha avanzado en la delimitación, vale decir, en la fijación del contenido y fronteras de esta nueva disciplina del derecho público, aún en desarrollo.

El derecho procesal constitucional hoy ha recibido aportes significativos de académicos provenientes del derecho procesal y del derecho constitucional, estudiando en profundidad las materias de confluencia disciplinaria, además del convencimiento de dotar tal estudio de autonomía como disciplina científica, en torno al cual se ha generado y desarrollado el Instituto Iberoamericano de Derecho Procesal Constitucional y sus nacientes ramas nacionales desde M éxico hasta Argentina y Chile en el extremo austral, teniendo la oportunidad de presidir la organización del IV Encuentro Iberoamericano de Derecho Procesal Constitucional desarrollado en Santiago en 2006, habiéndose ya desarrollado el primero en Rosario, A rgentina, en 2003; el segundo en San José, Costa Rica, en 2004; el tercero, en La Antigua, Guatemala, 2005; y el quinto, en Cancún, M éxico, en 2008, celebrándose el sexto en San Juan, Argentina, en septiembre de 2009.

17 Zagrebelsky, Gustavo. ¿Derecho Procesal Constitucional? y otros ensayos de justicia constitucional. Ed. FUNDAp, México, 2004, p. 57. 
En este homenaje al maestro Fix-Zamudio, por parte de la academia chilena concurren procesalistas, constitucionalistas y cultores del derecho internacional de los derechos humanos, rama del derecho esta última tan interrelacionada con los derechos fundamentales y que otorga una garantía inter o supranacional complementaria de la protección nacional de ellos. Esta confluencia de estudios y disciplinas jurídicas es lo que el maestro Fix-Zamudio busca y potencia al sostener que el derecho procesal constitucional se descompone en una dimensión de jurisdicción constitucional orgánica, una jurisdicción constitucional de la los derechos fundamentales y una jurisdicción constitucional trasnacional e internacional.

Los artículos recopilados en el presente libro son una muestra de la confluencia de los cultores de estas tres dimensiones del derecho procesal constitucional, los trabajos sobre las garantías del derecho a la jurisdicción y al debido proceso, el análisis sobre los órganos de jurisdicción constitucional y sus competencias, los procesos constitucionales $y$, en especial, del nuevo recurso de inaplicabilidad por inconstitucionalidad del artículo $93 \mathrm{~N}^{\circ} 6$ de la Constitución y sus peculiaridades, las acciones protectoras de derechos fundamentales, la Corte Interamericana y la protección de los derechos humanos, sin perjuicio de los temas relativamente nuevos del ministerio público en el proceso acusatorio penal, como el tema de la globalización y los derechos humanos, son analizados con diversos matices por calificados cultores académicos del derecho público constitucional, procesal e internacional, ellos son en el ámbito del derecho procesal, partiendo por el distinguido Presidente del Tribunal Constitucional, Juan Colombo, los Ministros José Luis Cea, Enrique Navarro y M arisol Peña; la Presidenta de la Corte Interamericana de Derechos Humanos, Cecilia Medina; los profesores de Derecho Procesal Andrés Bordalí, M iguel Otero, Diego Palomo y Hugo Pereira; los profesores de Derecho Constitucional Lautaro Ríos, Francisco Zúñiga y Humberto Nogueira.

El primer artículo del texto, del profesor Andrés Bordalí, se dedica al estudio de la defensa jurisdiccional de la Constitución y las instituciones de la justicia constitucional, relevando en un análisis crítico los temas de la difusión o concentración del control de constitucionalidad, la interpretación de la constitución por parte de todos los jueces, las sentencias interpretativas del Tribunal Constitucional y la casación dentro de las competencias de la Corte Suprema cuando ella se produce por motivos de inconstitucionalidad.

El Profesor y ministro del Tribunal Constitucional, José Luis Cea, analiza en su artículo los nuevos desafíos del Tribunal Constitucional chileno, luego de la reforma de 2005, centrando su trabajo en el nuevo $96 \mathrm{~N}^{\circ} 3$ sobre inaplicabilidad por inconstitucionalidad de leyes en control concreto y efecto inter partes, como asimismo analizando los criterios emplead os por el tribunal, además de sus propios criterios, sobre aspectos de procedimiento, como son la admisibilidad y el proceso de inaplicabilidad, teniendo presente la necesaria regulación legal de tales aspectos que concretará las reformas a la LOC del Tribunal, que hoy se encuentra en fase final de control de constitucionalidad preventiva por el propio Tribunal Constitucional. 
El Presidente del Tribunal Constitucional, Juan Colombo, realiza algunos alcances conceptuales en materia de derecho procesal constitucional y sus desafíos en el siglo XXI, para luego considerar la evolución del recurso de inaplicabilidad por inconstitucionalidad de las leyes, su transferencia como control concreto al Tribunal Constitucional desde la Corte Suprema de Justicia a partir de la reforma constitucional de 2005, como el desarrollo de sus consideraciones sobre su admisibilidad y tramitación.

El Ministro del Tribunal Constitucional, Enrique Navarro, centra su estudio sobre la reforma constitucional de 2005 en materia de jurisdicción constitucional, la nueva integración del Tribunal y sus nuevas competencias poniendo énfasis en el monopolio del control de constitucionalidad de la ley, para terminar con un análisis de la evolución del recurso de protección en materia de derechos fundamentales y una reflexión sobre los derechos no tutelados.

La Ministra del Tribunal Constitucional, Marisol Peña, pone su centro de atención en el artículo que nos proporciona, sobre las reformas al Tribunal Constitucional y su nueva competencia en materia de inaplicabilidad por inconstitucionalidad, como en los dilemas que debió enfrentar el Tribunal en el desarrollo del procedimiento sin que este estuviere establecido en la LOC del Tribunal Constitucional, analizando la jurisprudencia del tribunal producida sobre la materia.

El profesor Lautaro Ríos en su trabajo realiza una aproximación conceptual a la jurisdicción constitucional y nos presenta un panorama de ella en la evolución constitucional chilena, como asimismo cómo ella se encuentra distribuida en la competencia de diversos tribunales que tienen atribuciones específicas, para concluir con un análisis del Tribunal Constitucional y sus atribuciones actuales.

El profesor Humberto Nogueira desarrolla en su trabajo sobre el derecho a la jurisdicción y al debido proceso, él analiza el artículo $19 \mathrm{~N}^{\circ} 3$ y el artículo 76 de la Constitución en armonía con los artículos 8 y 25 de la Convención Americana sobre Derechos Humanos, como asimismo la rica jurisprudencia emanada del Tribunal Constitucional y de la Corte Interamericana de Derechos Humanos sobre la materia.

El profesor Diego Palomo realiza un análisis crítico sobre el Recurso de Protección en cuanto acción y proceso constitucional protector de los derechos fundamentales, analizando sus antecedentes, sus elementos esenciales, el desarrollo de la doctrina de la propietarización de los derechos y las perspectivas de cambio con el proyecto de ley de protección de derechos fundamentales en actual tramitación parlamentaria, habiendo ya sido aprobado por la Comisión de Derechos Humanos de la Cámara de Diputados en 2008.

El profesor Francisco Zúñiga analiza el tema del Fuero Parlamentario y las normas reguladoras del nuevo proceso penal, partiendo por un análisis de la naturaleza jurídica de la institución y su evolución en nuestro ordenamiento jurídico hasta llegar al actual procedimiento de desafuero parlamentario, considerando el desarrollo jurisprudencial del Tribunal Constitucional sobre la materia. 
Los profesores M iguel Otero y Hugo Pereira desarrollan dos estudios centrad os en el Ministerio Público como institución de la nueva justicia penal. El primer estudio explicita las diferencias entre el proyecto original del Ejecutivo y el texto aprobado por el Congreso Nacional respecto de las bases constitucionales del Ministerio Público; el segundo trabajo se centra en el análisis de las disposiciones constitucionales del Ministerio Público y su legislación complementaria, analizando las funciones y atribuciones del M inisterio público en el nuevo procedimiento penal acusatorio.

La profesora de Derecho Internacional de los Derechos Humanos y Presidenta de la Corte Interamericana de Derechos Humanos, Cecilia M edina, considera en su trabajo la jurisprudencia de la Corte Interamericana la calidad de víctima de los familiares de las personas que han sido víctimas de la violación de sus derechos fundamentales, especialmente considerando el derecho a la jurisdicción y al debido proceso, asegurados y garantizados por el artículo $8^{\circ}$, y el derecho a un recurso sencillo, rápido y eficaz para la protección de los derechos garantizados por el artículo 25 de la Convención Americana sobre Derechos Humanos.

Esperamos que los diversos trabajos recopilados en este libro homenaje al maestro Héctor Fix-Zamudio contribuyan a profundizar la reflexión de los cultores del derecho procesal y constitucional, como asimismo, a incentivar nuevos estudios e investigaciones en el campo del derecho procesal constitucional.

El libro contiene dos trabajos de profesores chilenos radicados en México que trabajan en la UNAM, Jorge Witker y Luis Díaz. El primero trata el tema de derechos humanos, derecho de competencia y garantía de los consumidores, realizando un análisis de los efectos horizontales y verticales de los derechos humanos. El segundo, centra sus reflexiones sobre el tema de la globalización como fenómeno, los desafíos de la ideología globalizadora en el ámbito cultural de los Estados y de Latinoamérica, como asimismo los desafíos de la globalización de los derechos humanos.

Al terminar esta presentación del texto, debemos agradecer a nuestro apreciado amigo y Presidente del Tribunal Constitucional y a las señoras y señores ministros, el que hayan prestado su casa como anfitriones para la presentación de la presente obra, ello demuestra una vez más la preocupación de nuestro Tribunal Constitucional, además de su tarea jurisdiccional, por estar presente en el desarrollo de la cultura jurídica nacional y del desarrollo y análisis de los temas académicos que están vinculados directamente con sus importantes y delicadas competencias constitucionales.

HUM BERTO NOGUEIRA ALCALÁ

Presidente de la Asociación Chilena de Derecho Constitucional Vicepresidente del Instituto Iberoamericano de Derecho Procesal Constitucional M iembro A sociado de la A cademia Internacional de Derecho Comparado. 